\title{
A homozygous BMPR 1B mutation causes a new subtype of acromesomelic chondrodysplasia with genital anomalies
}

\author{
O Demirhan*, S Türkmen*, G C Schwabe, S Soyupak, E Akgül, D Taștemir, D Karahan, S Mundlos, \\ K Lehmann
}

We present a patient with acromesomelic chondrodysplasia and genital anomalies caused by a novel homozygous mutation in BMPRIB, the gene coding for bone morphogenetic protein receptor $1 \mathrm{~B}$. The 16 year old girl, the offspring of a multiconsanguinous family, showed a severe form of limb malformation consisting of aplasia of the fibula, severe brachydactyly, unar deviation of the hands, and fusion of carpal/tarsal bones. In addition, she presented with hypoplasia of the uterus and ovarian dysfunction resulting in hypergonadotrophic hypogonadism. Mutation analysis of BMPR 1B revealed a homozygous 8 bp deletion (del359366). This mutation is expected to result in a loss of function and is thus different from the heterozygous missense mutations in BMPRIB recently shown to cause brachydactyly type A2 through a dominant negative effect. The patient's skeletal phenotype shows an overlap with the clinical spectrum of the acromesomelic chondrodysplasias of the Grebe, HunterThompson, and DuPan types caused by homozygous mutations in the gene coding for growth differentiation factor 5 (GDF5) which is a high-affinity ligand to BMPR1B. However, the phenotype described here differs from GDF5 associated chondrodysplasias because of the additional presence of genital anomalies and the distinct limb phenotype.

O steochondrodysplasias are a clinically and genetically heterogeneous group of disorders. ${ }^{1}$ Acromesomelic chondrodysplasias are a rare subgroup of these hereditary skeletal disorders characterised by short stature, very short limbs, and hand/foot malformations. The severity of limb abnormalities increases from proximal to distal with profoundly affected hands and feet showing brachydactyly and/or rudimentary fingers (knob-like fingers). The most severe type of the acromesomelic chondrodysplasias is known as Grebe syndrome, followed by the milder phenotypes of Hunter-Thompson and DuPan syndromes. These three related disorders share an autosomal recessive inheritance pattern and are caused by homozygous mutations in growth differentiation factor 5 (GDF5). Heterozygous carriers of GDF5 mutations are usually affected by brachydactyly type C (BDC), ${ }^{2-4}$ a condition characterised by brachymesophalangy of index, middle, and little fingers and shortening of the first metacarpal. Ring fingers are only mildly affected and hyperphalangy of the index and middle fingers occurs. However, as shown recently, BDC can also be caused by a homozygous mutation in GDF5. ${ }^{5}$

GDF5 belongs to the bone morphogenetic proteins (BMPs) of the transforming growth factor- $\beta$ (TGF- $\beta$ ) superfamily. GDF5 plays an essential role in chondrocyte condensation and differentiation as well as in joint formation as demonstrated by the brachypodism (bp) mouse which carries a loss of function mutation in Gdf5..$^{-8}$ The limb phenotype of the bp mutant consists of aplasia/hypoplasia of the middle and proximal phalanges and the metacarpals/metatarsals. The murine phenotype thus resembles the human homozygous GDF5 mutations.

GDF5 signalling requires binding to the serine/threonine kinase type of bone morphogenetic protein receptors (BMPRs). Three BMPRs are known (BMPRIA, BMPR1B, BMPR2) that are able to form multimeric complexes at the cell surface. ${ }^{9}{ }^{10}$ Binding of the ligand results in dimerisation of the type 2 receptor with one of the type 1 receptors and its subsequent transphosphorylation. Activation of the receptor complex results in phosphorylation of Smads that translocate in the nucleus where they regulate the transcription of target genes. ${ }^{11}$

Inactivation of BmpRIb in the mouse results in severe brachydactyly similar to the bp mutant mouse. ${ }^{12}{ }^{13}$ In addition, female $B m p R l b^{-/-}$mice are infertile indicating that BmpRlb plays a major role in the female reproductive system. ${ }^{14}$ This has also been shown in other species, for example in Booroola Mérino sheep, in which a specific BmpRIb missense mutation (Q249R) increases the ovulation rate and results in twin and triplet births. ${ }^{15-17}$

Recently, we demonstrated that heterozygous missense mutations in BMPRIB cause autosomal dominant inherited brachydactyly A2 (BDA2), a condition characterised by short and lateral deviated index and little fingers and normal fertility. ${ }^{18}$ Here we present a patient with a novel homozygous mutation in BMPRIB who has severe acromesomelic dysplasia and ovarian dysfunction.

\section{METHODS}

\section{Case report}

Clinical and radiographic investigations were performed in the Department of Medical Biology and Genetics and the Department of Radiology of the Çukurova University in Adana, Turkey. Informed consent was obtained from all participating family members.

The subject, a 16 year old girl (II-2), belongs to a multiconsanguinous Turkish family; her parents are first cousins and she has four siblings (fig 1). She presented with disproportionate short stature (height: $127 \mathrm{~cm}$ ) due to severe acromesomelic limb shortening (fig 2A). The length of the upper arm was $26 \mathrm{~cm}(-2.4 \mathrm{SD})$ and the length of the forearm $16 \mathrm{~cm}(-5.4 \mathrm{SD})$. The trunk was of normal length. Her hands (fig 2B) showed severe brachydactyly and radially deviated fingers. All fingernails were present. Hand radiographs showed severely hypoplastic phalangeal bones I-V (fig $2 \mathrm{Cl}$, right hand; fig $2 \mathrm{C} 2$, left hand); the proximal phalanges of the index fingers were missing. The tuberosity

Abbreviations: BDA2, brachydactyly A2; BDC, brachydactyly type C; $B M P$, bone morphogenetic protein; $B M P R$, bone morphogenetic protein receptor; bp mouse, brachypodism mouse; FSH, follicle stimulating hormone; GDF5, growth differentiation factor 5; LH, luteinising hormone; MCPP, metacarpophalangeal profile; TGF- $\beta$, transforming growth factor- $\beta$ 
of the distal phalanges of the middle fingers was altered and appeared bifurcated. The metacarpals II-V were of normal length, but metacarpals I were rudimentary. Both thumbs consisted only of a distal phalanx and the proximal phalanges were missing (fig 2B). The carpal bones showed an abnormal configuration and several bones were fused. On the right hand, the trapezium, capitate, and scaphoid bones and the hamate, triquetral, and lunate bones were fused; only the pisiform bone was separated but dislocated. On the left hand, except for an abnormally shaped pisiform bone, all other carpal bones were fused. The ulna was short and the radius appeared broad with an expanded distal epiphysis. At the age of 16, the epiphysial plate of the ulna and the radius were not closed indicating retardation of bone age. The feet showed a bilateral clubfoot deformity with small broad feet and short toes that appeared constricted at their bases (fig 2E). The toe nails were present. Radiographs of the lower leg (fig $2 \mathrm{~F}$ ) displayed deformed tibiae with wide proximal metaphyses and aplasia of the fibulae resulting in bilateral dislocation of the tibiotalar joints. The tarsal bones were partly fused, but the metatarsals were not affected. A radiograph of the pelvis (fig $2 \mathrm{G}$ ) showed short femoral necks and deformed femoral heads with irregular joint spaces; the femoral growth plates were not completely closed. Clinical examination showed limited cervical spine movements, but no radiograph of the cervical vertebrae was obtained.

In addition to the skeletal phenotype, the patient presented with genital anomalies and primary amenorrhea. Upon ultrasound examination, the ovaries were not present and the uterus was shown to be hypoplastic. Endocrinological studies confirmed hypergonadotrophic hypogonadism indicating a disturbance in ovarian function, with luteinising hormone (LH) 32 IE/l (standard values in follicle maturation and luteal phase: 2-20 IE/l, mid-cycle peak: up to $100 \mathrm{IE} / \mathrm{l}$ ), follicle stimulating hormone (FSH) $80 \mathrm{IE} / \mathrm{l}$ (standard values in follicle maturation and luteal phase: $2-8$ IE/l, mid-cycle peak: up to $25 \mathrm{IE} / \mathrm{l}$, postmenopausal value: $20-100 \mathrm{IE} / \mathrm{l}$ ), and oestradiol $<13 \mathrm{pg} / \mathrm{ml}$ (cycle dependent values 30-350 pg/ml, postmenopausal value: $10-35 \mathrm{pg} / \mathrm{ml}$ ). The patient receives hormonal substitution therapy. The diagnostics of LH, FSH, and oestradiol in the patient's mother and two sisters revealed standard values according to their age. There was no family history of skeletal disorders. The height of the parents was $162 \mathrm{~cm}$ (father, 3rd centile for Turkish men) and $150 \mathrm{~cm}$ (mother, 10th centile for Turkish women). Clinical examination of the proband's parents and siblings showed no obvious anomalies of the hands or feet.

\section{Metacarpophalangeal profiles}

To test for minimal symptoms of brachydactyly, metacarpophalangeal profiles (MCPPs) of hand radiographs of family members were carried out. For analysis of MCPPs, the program "Antro-Radiological Anthropometry of the Hand" was used. Further information about this programme can be obtained from www.hosenfeld.de/friedel/antro/antromai. htm.

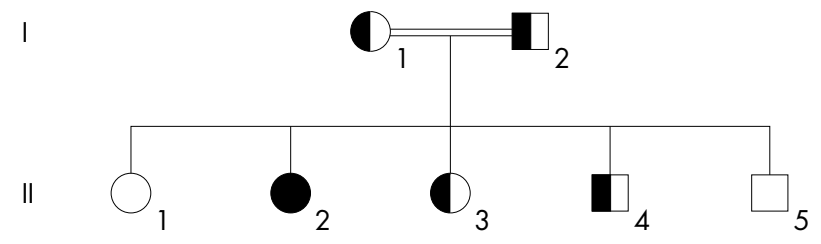

Figure 1 Pedigree. The patient is indicated by a filled symbol and heterozygous mutation carriers are indicated by semi-filled symbols. The parents are first cousins. There is no positive family history of skeletal disorders, infertility, or multiple pregnancies.
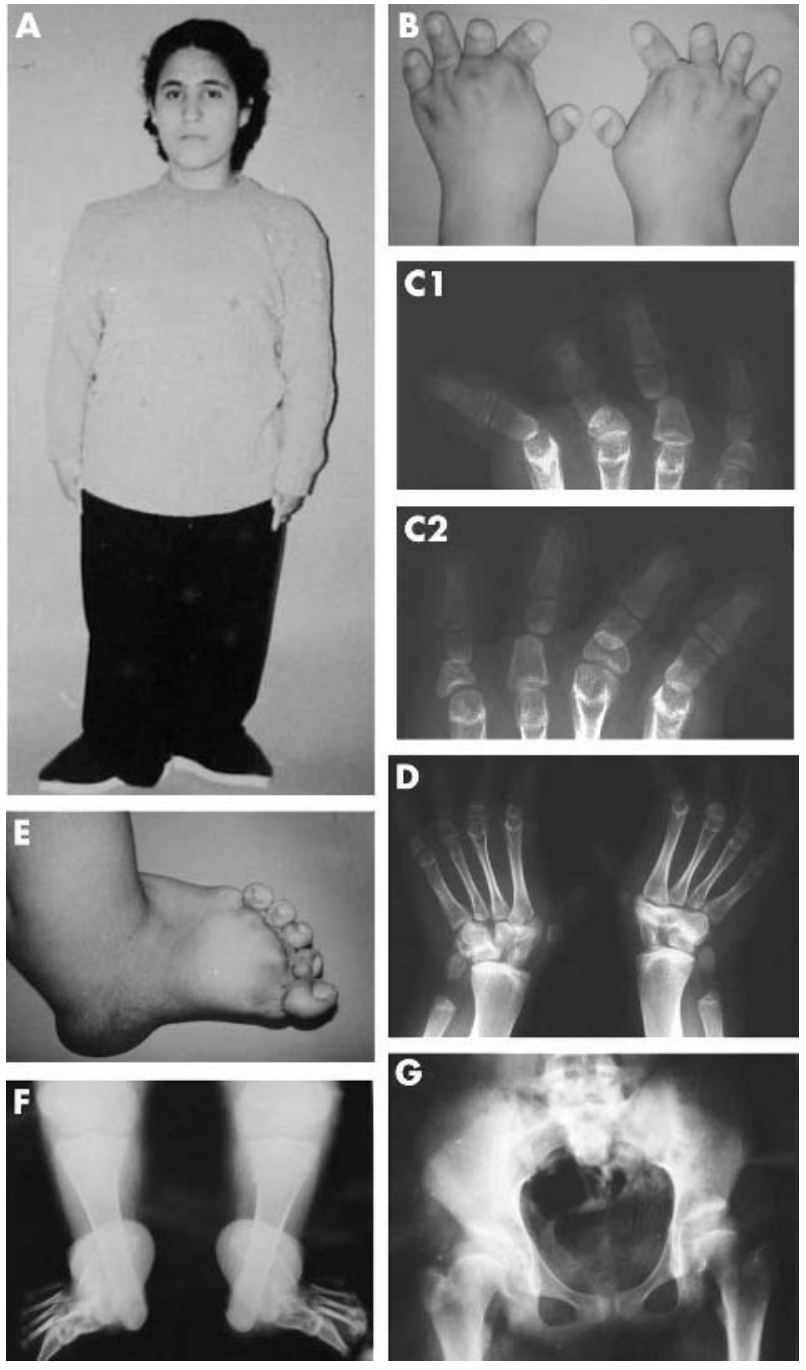

Figure 2 Phenotype. (A) Individual with a homozygous mutation in BMPRIB presenting with short stature and acromesomelic limb shortening. (B) The hands show severe brachydactyly of all digits. (C1, C2) The phalangeal bones II-V are hypoplastic and the proximal phalanges II are missing. (D) Metacarpals I are hypoplastic, but metacarpals II-V are of normal length. Fusion of carpal bones. (E, F) Clubfoot deformity of the feet due to an absent fibulae. The tibiae show broadened proximal metaphyses. (G) The femoral necks are short and the femoral heads are deformed. (Photograph reproduced with permission.)

\section{Molecular analysis}

Genomic DNA was extracted from peripheral blood samples by standard methods. The coding region of BMPRIB, consisting of 10 exons, was amplified by standard PCR protocols using a published primer set. ${ }^{18}$ PCR products were analysed on $2 \%$ agarose gels. Purified PCR products were sequenced in both directions using PCR primers as sequencing primers and the ABI Prism BigDye terminator cycle sequencing reaction kit (Applied Biosystems, Foster City, CA, USA). The products were evaluated on an ABI 3100 DNA sequencer (Applied Biosystems).

The entire coding region of GDF5 was also amplified and sequenced. Exact PCR conditions and primers are reported elsewhere. ${ }^{5}$

\section{RESULTS}

\section{Sequencing of GDF5 and BMPR $1 B$}

Because of the skeletal phenotype of acromesomelic chondrodysplasia in the patient, we first sequenced GDF5; a mutation in GDF5 was not found. 
Since the brachydactyly phenotypes associated with GDF5 and BMPRIB mutations show overlapping features, we then sequenced $B M P R I B$. We performed mutation screening of $B M P R I B$ in the affected girl, her parents, her two sisters, and her two brothers. In the patient, a homozygous 8 bp deletion (del359-366) in exon 4 of BMPRIB (fig 3) was identified. The mutation lies within the extracellular ligand binding domain of BMPRIB as shown in fig 4 and is predicted to result in a frame shift including the transmembranous and the entire intracellular domain of the receptor. Both parents and two siblings were heterozygous for the same mutation and two siblings were found to have both wt alleles.

\section{Metacarpophalangeal profiles}

MCPP analysis of hand radiographs demonstrated a discrete shortening (not exceeding the 2nd deviation in SD) of some distal phalanges in heterozygous mutation carriers I-2 and II3 and also in individual II- 1 who had two wt alleles. The lengths of metacarpals, and proximal and middle phalanges were within regular limits. In particular, the bones of the second ray affected in BDA2 were not altered in shape or length. Individual I-1, who is heterozygous for the BMPRIB mutation, showed a normal hand radiograph. These MCPP findings indicate that mutation carrier status is not associated with mild signs of brachydactyly.

\section{DISCUSSION}

In this report, we present a 16 year old girl with acromesomelic chondrodysplasia, genital anomalies, amenorrhea, and hypergonadotrophic hypogonadism due to a homozygous mutation in BMPRIB. The skeletal phenotype observed in this individual is distinct from the limb malformations described in other chondrodysplasias. The association of acromesomelic dysplasia with genital anomalies and ovarian dysfunction are novel and argue for a new subtype within the group of acromesomelic dysplasias. The mutation described here causes a frame shift in the region coding for the extracellular domain of BMPRIB and can thus be expected to result in a loss of function. BMPR1B is the major receptor for GDF5, ${ }^{19}$ a signalling molecule of the BMP family that plays a major role in digit formation, joint development, and chondrocyte differentiation. Mutations in GDF5 and mutations that result in a non-functional $B M P I B$ receptor, as in the case described here, can thus be expected to give rise to overlapping phenotypes.

The patient presented here shows many features overlapping with Grebe, Hunter-Thompson, and DuPan syndrome patients. In all conditions there is severe shortening of the fingers. Forearms and lower legs are severely shortened, the carpal/tarsal bones are deformed, and the metacarpals/
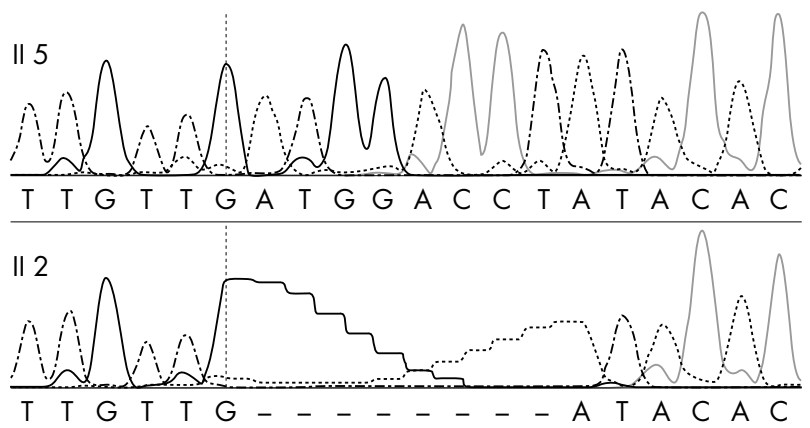

Figure 3 Sequence analysis of exon 4 in BMPRIB. Sequence of proband II-5 showing wild type sequence and sequence of affected individual II-2 showing homozygous 8 bp deletion (ATGGACCT) are shown. The deletion is expected to result in a frameshift and an early termination codon.
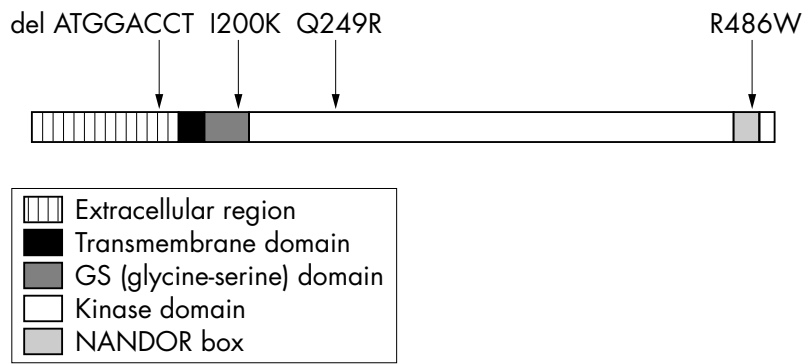

Figure 4 Domains and mutations in BMPRIB. A schematic of the BMP receptor $1 B$ is shown. BMPR IB is a serine/threonine receptor consisting of an extracellular domain, a transmembrane domain, and an

intracellular kinase domain. The homozygous 8 bp deletion (del359-

$366)$ reported here is located in the extracellular domain. Heterozygous amino acid changes $1200 \mathrm{~K}$ and R486W in humans cause BDA2.

Heterozygous and homozygous Q249R substitutions in Booroola Mérino sheep are associated with a higher ovulation rate and increased litter size.

metatarsals are short. In general, the condition described here is much less severe than Grebe syndrome and very similar to DuPan syndrome. Both DuPan syndrome and the phenotype described here have hypoplastic/absent fibulae and knob-like toes, and the hands are less severely affected than in Grebe syndrome. However, there are specific changes of the hand and foot bones that are unique and that distinguish the individual presented here from previously published cases. The metacarpal and metatarsal bones II-V are relatively normal, whereas in Hunter-Thompson and DuPan syndromes they are shortened to variable degrees. ${ }^{20-23}$ The extensive fusion of carpal/tarsal bones as observed in our patient is also not a typical feature of these conditions. $B m p R I b$ inactivation in the mouse results in a phenotype very similar to that presented here. BmpRIb ${ }^{-1-}$ mice display skeletal malformations that are restricted to the appendicular skeleton consisting of hypoplasia/aplasia of all phalanges and fusion of carpal/tarsal bones but relatively normal metacarpals/metatarsals. ${ }^{12} 1324$

The hands and feet of heterozygous BMPRIB mutation carriers in this family showed no signs of brachydactyly. MCPP analysis, a sensitive method to detect shortening of tubular hand bones, also showed no characteristic pattern for heterozygous mutation carriers. The lack of brachydactyly in heterozygous mutation carriers of a presumably loss of function mutation leads to the conclusion that one wt $B M P R I B$ allele is sufficient for proper bone formation in hands and feet. In contrast, heterozygous amino acid substitutions in the intracellular part of BMPRIB have been shown to cause BDA2 by acting in a dominant negative manner. ${ }^{18}$ Thus, specific missense mutations in BMPRIB result in a stronger effect than mere haploinsufficiency. In contrast, heterozygous carriers of loss of function mutations in the BMPRIB ligand GDF5 are affected with BDC. ${ }^{24}{ }^{25}$ The dominant character of these mutations indicates that GDF5 has important functions that are not transmitted via the BMPIB receptor and that other receptors are important in this particular phase of skeletal development. The dominant mechanism could also suggest that the ligand GDF5 is essential for proper skeletal development and that haploinsufficiency of GDF5 has a significantly stronger effect than haploinsufficiency of BMPRIB.

Female $B m p R I b^{-1-}$ mice are infertile due to irregular oestrous cycles, reduced quantity of cumulus cell-oocyte complexes in tertiary follicles, and a failure in proper uterine endometrial gland development. In vivo, oocytes of $B m p R I b^{-1-}$ mice cannot be fertilised as a result of disordered 
cell expansion of cumuluscells, which are necessary for effective sperm penetration. ${ }^{14}$ The importance of BMP signalling in ovarian function has been supported by the identification of mutations in ligands and receptors of the BMP pathway in sheep. A Q249R mutation in BmpRIb in Booroola Mérino sheep was shown to be associated with a higher ovulation rate and increased litter size. The mutation has an additive effect on reproduction with each mutation copy generating one or two extra lambs on average. ${ }^{15-17}$ The importance of the BMP pathway in the regulation of the ovulation rate is underlined by mutations in BMP15, another ligand of BMPRs, found in Inverdale and Hanna sheep. ${ }^{26}{ }^{27}$ In these strains, heterozygous mutations in BMP15 result in an increased ovulation rate, whereas homozygous mutations lead to small non-functional streak ovaries with primary ovarian failure and infertility.

Sheep with BMPRIB or BMP15 missense mutations do not show a skeletal phenotype. In contrast, $B m p R I b^{-/-}$mice caused by a kinase deletion in BmpRlb present with brachydactyly and infertility. ${ }^{14}$ Thus, the skeletal and genital phenotype of the BmpRIb $b^{-1-}$ mouse is similar to the clinical anomalies associated with the human BMPRIB mutation described in this report. The presence of acromesomelic dysplasia associated with ovarian and uterine disorder in the female patient described here highlights the dual function of BMPRIB in skeletal development as the predominant receptor for GDF5 on one hand and its role in genital development and ovarian function on the other. Further investigations of BMPRIB mutations and the characterisation of associated phenotypes will be of interest in understanding the exact biological function of BMPRIB in humans.

\section{ACKNOWLEDGEMENTS}

The authors thank the family members who participated in this study.

\section{ELECTRONIC-DATABASE INFORMATION}

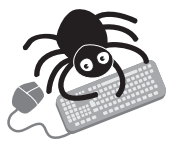

Information about the program "Antro-Radiological Anthropometry of the Hand" can be obtained from www.hosenfold.de/friedel/antro/antromai.htm

\section{Authors' affiliations \\ O Demirhan, D Taștemir, D Karahan, Department of Medical Biology and Genetics, Cukurova University, Adana, Turkey \\ S Türkmen, S Mundlos, K Lehmann, Institute for Medical Genetics, Charité, Berlin, Germany \\ G C Schwabe, S Mundlos, Max Planck Institute for Molecular Genetics, Berlin, Germany \\ S Soyupak, E Akgül, Department of Radiology, Çukurova University, Adana, Turkey \\ Competing interests: none declared \\ *Both authors contributed equally to this work.}

Correspondence to: Stefan Mundlos, Institut für Medizinische Genetik, Universitätsmedizin Berlin, Charité, Augustenburger Platz 1, 13353 Berlin, Germany; stefan.mundlos@charite.de

Revised version received 2 July 2004

Accepted for publication 6 July 2004

\section{REFERENCES}

1 Kornak U, Mundlos S. Genetic disorders of the skeleton: a developmental approach. Am J Hum Genet 2003;73(3):447-74

2 Everman DB, Bartels CF, Yang Y, Yanamandra N, Goodman FR, MendozaLondono JR, Savarirayan R, White SM, Graham JM Jr, Gale RP, Svarch E, Newman WG, Kleckers AR, Francomano CA, Govindaiah V, Singh L, Morrison S, Thomas JT, Warman ML. The mutational spectrum of brachydactyly type C. Am J Med Genet 2002;112(3):291-6.
3 Polinkovsky A, Robin NH, Thomas JT, Irons M, Lynn A, Goodman FR, Reardon W, Kant SG, Brunner HG, van der Burgt I, Chitayat D, McGaughran J, Donnai D, Luyten FP, Warman ML. Mutations in CDMP1 cause autosomal dominant brachydactyly type C. Nat Genet 1997; 17(1): 18-9.

4 Savarirayan R, White SM, Goodman FR, Graham JM Jr, Delatycki MB, Lachman RS, Rimoin DL, Everman DB, Warman ML. Broad phenotypic spectrum caused by an identical heterozygous CDMP-1 mutation in three unrelated families. Am J Med Genet 2003;117A(2):136-42

5 Schwabe GC, Turkmen S, Leschik G, Palanduz S, Stover B, Goecke TO, Mundlos $S$. Brachydactyly type $C$ caused by a homozygous missense mutation in the prodomain of CDMP1. Am J Med Genet 2004;124A(4):356-63.

6 Storm EE, Huynh TV, Copeland NG, Jenkins NA, Kingsley DM, Lee SJ. Limb alterations in brachypodism mice due to mutations in a new member of the TGF beta-superfamily. Nature 1994;368(6472):639-43.

7 Storm EE, Kingsley DM. GDF5 coordinates bone and joint formation during digit development. Dev Biol 1999:209(1):11-27.

8 Francis-West PH, Abdelfattah A, Chen P, Allen C, Parish J, Ladher R, Allen S, MacPherson S, Luyten FP, Archer CW. Mechanisms of GDF-5 action during skeletal development. Development 1999;126(6):1305-15.

9 Gilboa L, Nohe A, Geissendorfer T, Sebald W, Henis YI, Knaus P. Bone morphogenetic protein receptor complexes on the surface of live cells: a new oligomerization mode for serine/threonine kinase receptors. Mol Biol Cell 2000;11(3):1023-35.

10 Nohe A, Hassel S, Ehrlich M, Neubauer F, Sebald W, Henis YI, Knaus P. The mode of bone morphogenetic protein (BMP) receptor oligomerization determines different BMP-2 signaling pathways. J Biol Chem 2002;277(7):5330-8

11 Nohe A, Keating E, Knaus P, Petersen NO. Signal transduction of bone morphogenetic protein receptors. Cell Signal 2004;16(3):291-9.

12 Yi SE, Daluiski A, Pederson R, Rosen V, Lyons KM. The type I BMP receptor BMPRIB is required for chondrogenesis in the mouse limb. Development 2000;127(3):621-30.

13 Baur ST, Mai JJ, Dymecki SM. Combinatorial signaling through BMP receptor IB and GDF5: shaping of the distal mouse limb and the genetics of distal limb diversity. Development 2000;127(3):605-19.

14 Yi SE, LaPolt PS, Yoon BS, Chen JY-C, Lu JKH, Lyons KM. The type I BMP receptor BmprIB is essential for female reproductive function. Proc Natl Acad Sci U S A 2001;98(14):7994-9.

15 Mulsant P, Lecerf F, Fabre S, Schibler L, Monget P, Lanneluc I, Pisselet C, Riquet J, Monniaux D, Callebaut I, Cribiu E, Thimonier J, Teyssier, J, Bodin L, Cognie Y, Chitour N, Elsen J-M. Mutation in bone morphogenetic protein receptor-IB is associated with increased ovulation rate in Booroola Merino ewes. Proc Natl Acad Sci U S A 2001;98(9):5104-9.

16 Wilson T, Wu XY, Juengel JL, Ross IK, Lumsden JM, Lord EA, Dodds KG Walling GA, McEwan JC, O'Connell AR, McNatty KP, Montgomery GW. Highly prolific Booroola sheep have a mutation in the intracellular kinase domain of bone morphogenetic protein IB receptor (ALK-6) that is expressed in both oocytes and granulosa cells. Biol Reprod 2001;64(4):1225-35.

17 Souza CJ, MacDougall C, Campbell BK, McNeilly AS, Baird DT. The Booroola $(\mathrm{FecB})$ phenotype is associated with a mutation in the bone morphogenetic receptor type 1 B (BMPR1B) gene. J Endocrinol 2001;169(2):R1-6.

18 Lehmann K, Seemann P, Stricker S, Sammar M, Meyer B, Suring K, Majewski F, Tinschert S, Grzeschik KH, Muller D, Knaus P, Nurnberg P, Mundlos S. Mutations in bone morphogenetic protein receptor 1B cause brachydactyly type A2. Proc Natl Acad Sci U S A 2003;100(21): 12277-82.

19 Nishitoh H, Ichijo H, Kimura M, Matsumoto T, Makishima F, Yamaguchi A, Yamashita H, Enomoto S, Miyazono K. Identification of type I and type II serine/threonine kinase receptors for growth/differentiation factor-5. J Biol Chem 1996;271(35):21345-52.

20 Ahmad M, Abbas H, Wahab A, Haque S. Fibular hypoplasia and complex brachydactyly (Du Pan syndrome) in an inbred Pakistani kindred. Am J Med Genet 1990;36(3):292-6.

21 Langer LO Jr, Cervenka J, Camargo M. A severe autosomal recessive acromesomelic dysplasia, the Hunter-Thompson type, and comparison with the Grebe type. Hum Genet 1989;81(4):323-8.

22 Thomas JT, Lin K, Nandedkar M, Camargo M, Cervenka J, Luyten FP. A human chondrodysplasia due to a mutation in a TGF-beta superfamily member. Nat Genet 1996;12(3):315-7.

23 Faiyaz-Ul-Haque M, Ahmad W, Zaidi SH, Haque S, Teebi AS, Ahmad M, Cohn DH, Tsui LC. Mutation in the cartilage-derived morphogenetic protein(CDMP1) gene in a kindred affected with fibular hypoplasia and complex brachydactyly (DuPan syndrome). Clin Genet 2002;61(6):454-8.

24 Zhao M, Harris SE, Horn D, Geng Z, Nishimura R, Mundy GR, Chen D. Bone morphogenetic protein receptor signaling is necessary for normal murine postnatal bone formation. J Cell Biol 2002;157(6):1049-60.

25 Thomas JT, Kilpatrick MW, Lin K, Erlacher L, Lembessis P, Costa T, Tsipouras P, Luyten FP. Disruption of human limb morphogenesis by a dominant negative mutation in CDMP1. Nat Genet 1997;17(1):58-64.

26 Galloway SM, McNatty KP, Cambridge LM, Laitinen MP, Juengel JL, Jokiranta TS, McLaren RJ, Luiro K, Dodds KG, Montgomery GW, Beattie AE, Davis $\mathrm{GH}$, Ritvos $\mathrm{O}$. Mutations in an oocyte-derived growth factor gene (BMP15) cause increased ovulation rate and infertility in a dosage-sensitive manner. Nat Genet 2000;25(3):279-83.

27 Hanrahan JP, Gregan SM, Mulsant P, Mullen M, Davis GH, Powell R, Galloway SM. Mutations in the genes for oocyte-derived growth factors GDF9 and BMP15 are associated with both increased ovulation rate and sterility in Cambridge and Belclare sheep (Ovis aries). Biol Reprod 2004;70(4):900-9. 